\title{
From nuclear to meso systems: how small is simple and how large is complex $?^{\star, \star \star}$
}

\author{
Jean-Patrick Connerade ${ }^{1,2, \text { a }}$ \\ 1 Quantum Optics and Laser Science Group, Physics Department, Imperial College, Prince Consort Road, \\ London SW7 2BW, UK \\ 2 European Academy Sciences Arts Letters, EASAL, 60 rue Monsieur-le-Prince, 75006 Paris, France
}

Received 3 January 2017

Published online 25 April 2017

(c) The Author(s) 2017. This article is published with open access at Springerlink.com

\begin{abstract}
The question is asked: what are new size-dependent effects characteristic of the nanoscale range which justify considering 'dynamics on the nanoscale' as a scientific area in its own right rather than merely an extension of existing knowledge to a different parameter range. A preliminary list of such effects is drawn up. It is shown to include many challenging unsolved problems as well as a number of fundamental investigations which are more or less advanced in development at the present time.
\end{abstract}

\section{Introduction}

Nano-physics and, more generally, the nano-sciences are new areas of research which encompass several related fields, extending from molecular and cluster physics to novel materials and including also the study of certain types of biological system. Usually, the subject is approached from the perspective of its applications because they are so numerous and important, but the question does arise: is nanoscience a new field in its own right, or simply an extension of what was known previously on a different scale?

Behind this question is the thought that defining a field entirely from its applications is not really sufficient to determine a genuine subject area. More fundamentally, within the conceptual framework of physics, one thinks first of quantum mechanics for so-called 'microscopic systems' (meaning those of atomic size or smaller) and of classical mechanics for allegedly 'large' systems and there seems to be no room in principle for anything else different in between. So, the question posed here is really whether the words 'nano' and 'meso' refer simply to an intermediate scale of size or whether there are indeed new physical effects in this range which justify considering the nanosciences as a new and different field, i.e. a true subject with its own specific effects and emblematic quests.

In part, an answer to this question is implicit, since quantum physics, molecular dynamics, cluster and solid state physics, molecular biology, etc. all congregate around

\footnotetext{
* Contribution to the Topical Issue "Dynamics of Systems at the Nanoscale", edited by Andrey Solov'yov and Andrei Korol.

** Invited article based on discussion presentation at DYSON Conference, Bad Ems, 3-7 October 2016.

a e-mail: jean-patrick@connerade.com
}

systems on this scale. An interface between such a formidable array of different research areas, each with its specific array of methods, obviously defines an important boundary. However, what one is really looking for here is a catalogue of phenomena characteristic of nano-science, intimately related to the range of sizes involved in the DYSON meetings.

The concept of 'size' is in principle one of the simplest measures one can imagine. In quantum mechanics, length is a well-defined operator, and we can also think of an experimentally defined size, the scale of which is determined by the diameter of an atom of Hydrogen, i.e. by the atomic unit of length. Thus far, everything is simple. Life becomes a little more complicated (as will be described) when we ask what we actually wish to measure, because (as is usual in quantum mechanics) the method of measurement is crucial and must also be taken into account in order to decide what is 'large' and what is properly regarded as 'small' in a given physical system. Finally, we will consider the emergence of temperature and entropy, which can also be regarded as markers for the emergence of the nanoscale.

In order to address the issue raised in the title of this review, we need to consider where nano-science 'fits' in between systems smaller than atoms (nuclear physics) and systems of a size larger than 'nano' (the meso-scale). In practice, for the purpose of this review, we take the nanoscale as extending from one to several hundred nanometres and the meso-scale to extend beyong the nanoscale up to the sizes of large clusters or large biological molecules.

The purpose of the present review is NOT to recapitulate examples of well-documented effects which can be understood simply by extending principles valid for other scale sizes into the nano-range. Rather, the intention 
is to identify issues which are either specific to the nanorange or which arise differently from any other context, so that we can regard them as characteristic of a new area. We begin by considering 'size' in the quantum regime and then turn to a catalogue of effects which arise on the 'nano' to 'meso' scale.

\section{Size and scattering length in nuclear physics}

One of the best examples of a determination of size by direct measurement in a quantum system occurs in particle scattering and is exemplified in Nuclear Physics. The purpose of the experiment is to probe the structure (the interaction potential) of the scattering centre by observing the distribution and/or energy spectrum of the scattered particles. This problem, sometimes termed 'inverse scattering' because it implies an unfolding of the observed spectrum to determine the scattering potential, is treated in some detail in a number of textbooks (e.g. [1]) so we recall only the essential points.

The key to understanding the issues involved is the reflect on the nature of the scattering potential, not only in nuclear physics, but in any scattering problem. There are two possibilities. Either the potential falls off slowly with distance (at or more slowly than $1 / r$ ) in which case the potential is long range, or else it falls off faster than $1 / r$, in which case we speak of a short range potential (the Yukawa potential, for example). For a short range potential, there is a characteristic size called the scattering length $\left(\lambda_{\mathrm{s}}\right)$ which basically measures how big a target appears to the incoming particle. To measure the structure of a scattering centre and deduce its internal properties correctly, it is necessary that the de Broglie wavelength of the scattered particle $\left(\lambda_{\mathrm{DB}}=h / p\right)$ should be much smaller than $\lambda_{\mathrm{s}}$. This can only be achieved by increasing the momentum of the scattered particle used as a probe (which is the basic motivation for building accelerators). The penalty is of course that if the energy of the scattered particle becomes too high, then ultimately it will fragment the target. Thus, whether a system is 'small' in this context simply means that it is smaller than the $\lambda_{\mathrm{DB}}$ we can reasonably apply with a scattered particle in order to probe the structure of the short-range scattering potential.

This problem really illustrates quantum mechanics at work: the length which can actually be measured depends on what energy the observer can use for his probe (exactly as in the gamma ray microscope of Heisenberg [2], when we use high energy photons instead of material particles to measure size) and so a target becomes 'small' basically when the inversion of the scattered spectrum can yield no further information. Schwinger [3] allegedly took this argument one step further by remarking that any shape of short range potential of equivalent strength and scattering length would become equivalent to any other for scattering lengths down below this size, so that no information about the shape of the potential (apart from the scattering length itself) could be deduced from the low en- ergy scattering spectrum in this case. This was his basic motivation for setting up effective range theory.

At the opposite end of the size scale, we may ask about a long-range potential. In principle, the structure of such a potential would be readily observable, but such a potential, being very 'soft' has no specific length we can associate with the scattering. The best approach in this case is to consider the total scattering cross section and treat it as $\sigma_{\mathrm{TOT}}=\pi r_{\mathrm{s}}^{2}$, in which case $r_{\mathrm{s}}$ provides the simplest measure of effective size for such a 'large' system.

Thus, 'size' in Quantum Mechanics is a rather more complex issue than appears at first sight, because of what is basically a consequence of the uncertainty principle: it depends on the nature of the interaction with a probe particle and on its de Broglie wavelength in an actual experiment, not simply on the target itself. Quantum Mechanics makes physicists deeply aware of the importance of size. This idea was handed down to us by Niels Bohr, who believed what was then called 'microphysics' to be essentially different from classical physics and even that our scientific intuition about it would often be deceptive, because our sensorial experience is by nature based on the properties of classical systems [4].

\section{3 'Smallness' and classical physics}

I would further like to argue that 'smallness' as a fundamental property (the barrier between what we can see and what we can't) is a fundamental quantum property and therefore that 'smallness' does not even exist as such in classical physics.

This argument can be based on a famous text by the French scientist and philosopher Blaise Pascal [5], which sets out rather clearly the point of view of a classical physicist (or rather 'natural philosopher'). The context is that of a discovery by Dutch scientists who had just perfected the microscope and, having observed some very tiny creatures, declared them to be the smallest possible structures. Pascal was deeply opposed to this idea. He remarks that, within these creatures, there must be smaller parts and, within these parts, there must be atoms. But each of these atoms, in his view, is like a miniature solar system, with its planets and, within these, still more even smaller creatures, and so on ad infinitum, leading to a classical theory of a never-ending sequence of 'worlds within other worlds' (sometimes called Pascal's vertigo) with the apparent consequence that there is no minimum or maximum size for anything.

This theory would apply, not only to material objects, but would extend also to empty space, which thus becomes infinitely divisible (i.e. continuous) as in Einstein's theory of Relativity [6].

Even before the advent of Quantum Mechanics, it was becoming clear (mainly through the work of Gibbs [7]) that this infinite 'depth' of space and of matter could not exist. A key point is stressed by Dirac in his book on the Principles of Quantum Mechanics [8], in which he points out that, if classical physics (by which he understands something close to Pascal's description) were correct, 
the Equipartition Theorem of thermodynamics would lead to all specific heats being infinite. The fact that they are finite is one of the many fundamental reasons for believing in the quantum model, which sets a minimum 'size' for observable effects. Indeed, this relates to a very fundamental problem in contemporary physics: how can one reconcile the continuous space apparently required by Relativity (excepting perhaps some alternatives, explored by Célérier and Nottale [9] and others [10]) with the 'granular' structure of space and matter implicit in Quantum theory? This dichotomy of modern physics is both troubling and unresolved. I return to equipartition and size below.

\section{The correspondence principle and the issue of 'Quantum Chaos'}

The Correspondence Principle attempts to bridge the gap between Quantum Mechanics and Classical Physics and so it covers the full range of sizes from the ground state of an atom to a (somewhat hypothetical) 'giant Rydberg atom' which would obey rules obtained from Classical Mechanics and is referred to as the 'classical limit'. Therefore, it encompasses the nano- and meso-scales.

The connection between classical and quantum systems works pretty well if the classical dynamics of the system considered are fully integrable (closed classical orbits). In this case, one just applies the Bohr-Sommerfeld quantisation rule

$$
\int p d q=(n+1 / 2) \hbar \omega
$$

where the integration is carried out over a closed orbit for conjugate classical variables $p$ and $q$ and $n$ is the principal quantum number.

However, as first pointed out by Einstein [11], there is a problem in applying this rule if the orbits do not close. Orbits which do not close are in fact very common in classical mechanics, as discovered by Poincaré [12] for the three-body problem. They even occur for fairly simple situations, such as the classical pendulum above a magnet. By attaching a pen to the pendulum, so that it writes on a sheet of paper, one can verify that the pendulum will never retrace its steps, i.e. that within the limits defined by the total energy of the motion, the pendulum will 'write' all over the surface without the orbit ever closing.

The quantum analogue of this classical system is a Rydberg atom in a strong magnetic field, and there is an extensive literature, both experimental and theoretical, on the transition from quantum to 'classical' behaviour in atomic systems since the original experiments performed by Garton and Tomkins [13] first drew attention to this situation.

The real question posed here is complementary to the one posed by Einstein. He asked: how do you quantise a classically irregular system, but one can also ask the opposite question: in general, when one takes a quantum system to its classical limit, does one recover all of classical physics or only a part? In other words, do classically chaotic orbits emerge spontaneously in this limit, or does one recover only an incomplete form of classical mechanics?

Originally, it was hoped that a detailed experimental study of this inverse situation might help to resolve the long-standing issue raised by Einstein [11]. However, this is unfortunately not the case: indeed, for a quantum system whose underlying dynamics are chaotic, one observes experimentally the emergence of a great structural complexity in a spectral range corresponding to this limit. It is sometimes referred to (rather loosely) as a region of 'quantum chaos', but in reality, there is no hard evidence that it is truly 'chaotic' in the classical sense. Indeed, there are good reasons to assert that 'chaos' cannot arise at all in a quantum system. The argument runs as follows.

If we consider a classically chaotic system (for example, the pendulum and magnet) we expect the pen of the pendulum to write all over the paper within the boundaries defined by the conservation of energy, or (expressed more precisely) we expect the system to travel densely through all accessible regions of phase space. In other words, there should be no accessible region of phase space which contains no orbit. In order to check this, we must increase the magnification as far as we can and make sure that we always find a density of paths which are distinguishable from each other. If the orbit (whatever its complexity) eventually 'closes', then it is not chaotic. However, performing this test would in itself violate Quantum Mechanics: if at some point the orbits approach each other to within a volume $\hbar^{\mathrm{N}}$ in phase space, then we bump into what one might term a fundamental granularity of Quantum Physics: we are not allowed to ask what happens within such a small volume and so the question: is such an orbit chaotic or is it not? No longer has any meaning.

This is a very fundamental issue in terms of our understanding of quantum physics, and it seems fair to say that it is unresolved. It connects with the fundamental dichotomy between the two great theories of modern physics: quantum physics and relativity. Many attempts have been made to 'join' one theory onto the other, but the fact is that the nature of space-time in quantum theory is not the same as it is in relativity, precisely because of the 'granularity' referred to above.

The range of sizes within which such discrepancies become important is the nanoscale range. Unfortunately, no experiments so far have been able to uncover clear evidence of the failing of one or other approach to the interpretation, the most complete test being probably the study of Rydberg atoms in crossed electric and magnetic fields with full separation of circular polarisations, performed at Imperial College $[14,15]$ and analysed at the Queens University, Belfast $[16,17]$. In short, issues relating to the field of 'Quantum Chaology' belong to the subject of 'Dynamics at the Nanoscale' (DYSON).

\section{Order to many-body chaos}

Another and rather different kind of Order-to-Chaos transition appears, not as a result of a perturbation by an 
external field (as in the previous example) but as an intrinsic property of a many-body system. Instead of tending towards chaos in the classical limit, such a system, on the contrary, is subject to underlying chaotic forces when united but separates into two systems connected by regular forces when one particle is progressively removed from all the others. This type of system exists because few-body systems (starting with three-body systems) are classically chaotic, as proved by Poincaré [12]. They can only be quantised by invoking a fictitious 'independent particle, mean field theory', which is subsequently refined by introducing correlations in order to bring the theoretical results more in line with experiment. In this context, 'small' systems are considered to be 'simple' while 'large' ones are treated as 'complex'. However, one should bear in mind that symmetry also plays a role. For example, spherical closed shells exist in atoms, in certain molecules and even in metallic clusters, which can restore the meanfield approximation and 'simplify' even the treatment of a fairly large system. In atomic, molecular and cluster physics, many-body chaos is successfully treated perturbatively through correlations, because electrons are very prompt and the mean-field approximation works surprisingly well even for rather distorted systems.

In this context, note that 'size' no longer has the dimension of 'length'. Rather, it refers to the number of particles in the system. This is the usual meaning given to the 'size' of a system in nanoscale physics. It is used to discuss a number of size-related transitions, as listed below. In this picture, the atoms are regarded as basically incompressible, so that adding atoms to a system necessarily increases its size. At normal pressures, this will broadly be true and turns out to yield a very convenient size parameter.

\section{The What is life? transition}

It is a simple observational fact that 'living' systems always contain very many atoms or molecules. The smallest living system (a virus) can contain roughly one thousand atoms [18]. This raises the interesting challenge: is there a fundamental size below which life becomes impossible and (therefore) a fundamental reason for which this should be the case?

When we observe a living system in a favourable environment, it will send us, by replicating itself, a definite signal that it is alive. If we count the atoms (or molecules) in such a system, we have an estimate of its size. So, it is a meaningful scientific question to ask what connection exists between size and viability for a living system.

Schrödinger, in his famous essay What is Life? [19] asks a related question, which he formulates rather differently: why are atoms so small? i.e. why are organisms so large compared to atoms? The answer he suggests is that life requires exact physical laws. Without enough atoms in a system, statistical fluctuations become too large for life to develop. This is not a very precise answer, but seems to set a lower bound.
Notice also that this issue of life and size is tied in with Blaise Pascal's discussion referred to above [5]. On the one hand, the Dutch scientists whose work he objected to believed they had found the smallest living beings thanks to their microscope, but on the other, Pascal [5] was arguing that, according to the laws of science as understood in classical physics, there was no reason for the existence of any lower bound.

The question of the smallest living cell has interested NASA in connection with planetary exploration. Very small organisms appear in ground water after exhaustive filtration and pose questions as to their origin [20]. Amongst know germs, Mycoplasma and Rickettsia are about $100 \mathrm{~nm}$ in size and qualify as small. Mycoplasma do replicate as they are a bacteria, not a virus. They depend on a host (or rich growth medium in a lab) for nutrients they need in order to survive. Not all mycoplasma species invade a host cell: some can live outside of the host cell and can be grown in a rich growth medium as opposed to a cell culture [21].

One can also ask the opposite question, i.e. what is the largest living cell? The largest in volume is said to be the egg of an Ostrich. In linear dimension, the human body, for example, has neurons running from the base of the spine to our toes. These are the longest cells in the human body, some 60 to $90 \mathrm{~cm}$ long. However, they are still microscopically thick and depend on other cells to assist them by providing nutrients. So, one can argue about the relevance of such answers depending on the degree of autonomy expected of a living organism.

Nonetheless, what is clear is that the range of sizes which corresponds best to the possible transition in viability between inert and living systems falls in the nanoscale to mesoscale range. Consequently, to modernise and update Schrödinger's original question involves research in size-dependent biology relating to what is called 'nano-science'. Indeed, the observation has already been made [22] that an inert cluster and a living virus can be of comparable size, which in itself raises interesting issues about the difference between them both.

\section{Chaos and complexity}

Often, scientific activity involving large molecular systems is justified on the basis of attempting to come to grips with 'complexity'. The difficulty with this statement is trying to understand precisely what is meant by 'complexity'. Intuitively, one would expect that chaos might be present at the same time (or for similar systems) as complexity. However, one must be careful to distinguish mathematically between what may be very intricate and very complex and what is chaotic. Again, the distinction hinges on the closure of orbits discussed above. If there is no 'chaos' in Quantum Mechanics, then complexity may replace it, but how should it be defined? One can perhaps seek procedures to regularise orbits which would be chaotic in classical physics (for example, by considering how they are bundled according to the KAM theorem [23,24]) and describe these as 'complex', but if the definition remains ad hoc 
it can have little scientific value. Thus, although there is justification for the argument that, in the nanoscale range, one has to chose the 'best' between different approximations in order to calculate very complex situations, such a justification is based rather on the limitations of existing theory than on any new effect one might uncover. If exploring complexity is to serve as a motivation, then it will first need to be defined rather precisely in relation to the nanoscale range.

\section{The atom to solid transition}

The atom-to-solid transition was frequently described in many models used to set up the subject described as 'condensed matter' or 'solid state physics' and this description remained unchallenged for many years. Indeed, it found its way into a variety of textbooks, more or less as a conceptual model of how a solid is built up atom by atom, the simplest picture being a long linear chain. Leading contributions in developing this picture were made by Debye [25] (see e.g. [26]), Einstein [27], Blackman [28] and many others.

While this description is of intellectual value in picturing the nature of the solid state, recent researches in nanophysics have demonstrated very clearly that the original models as described in many textbooks are much oversimplified. Of course, what had not been expected is that one might be able one day to 'build up' a solid experimentally, i.e. to construct size-selected clusters composed of many atoms and to 'dial' the size of such systems between just a few (the atomic of molecular limit) and many thousands (the solid-state limit). It was therefore no longer a purely intellectual exercise to imagine how a solid might progressively be built up. The experiment became a real one.

Furthermore, with the advent of these new techniques and the birth of cluster physics, it became clear that one could track different physical quantities (speed of sound, conductivity, magnetism, optical properties, etc.) as a function of the size of the cluster, thus opening up new directions in the study to the atom-to-solid transition as a function of the number of atoms in the cluster, the type of atom, the kind of solid, etc.

On the way of these studies, new effects were discovered which had not been suspected before, such as the formation of closed shells consisting of many atoms for alkali or conducting clusters [29] (with the discovery of associated optical 'giant resonances' [30]), the discovery of magic numbers also for insulating and van der Waals clusters [31], etc. Without going into great detail about all the new and interesting effects, the main outcome of this research has been to re-write the material present in the opening chapters of the 'old' textbooks on solid state physics.

It is now understood that there is not one, but there are many transitions from the atomic to the solid state limits even for a solid built up from a single species. In fact, there are as many points of transition as there are physical parameters used to 'track' the evolution from one limit to the other or, put another way, the transition occurs for different sizes according to what property one is measuring. Also, each species (each type of atom) has its own form of evolution, which may bear similarities within a family of atoms, but remains unique as to the detail. For a review concerning such transitions, see [32].

All of this is now better understood thanks to nanophysics. Indeed, this is a fundamentally new contribution which has come from this area of research and falls very clearly within the scope of this particular area of research.

\section{Faraday screening on the nanoscale}

The endohedral encapsulation of atoms and molecules such as occurs in metallofullerenes, in 'Russian doll' molecules, 'peapod' nanotubes, etc. is an effect associated with the nanoscale and connected with a variety of potential applications. Since many of the molecules and surfaces involved (fullerenes and graphene, for example) are good conductors, the question arises: can such surfaces produce a 'Faraday cage' on the nanoscale? If so, then there would be numerous applications. For example, an atom placed at any position inside such a 'cage' would be isolated electrically from the world outside. This would imply, as first suggested by Harneit [33] that an encapsulated atom with intrinsic spin could be oriented in a given direction and would subsequently retain this orientation. Thus, a 'string' of encapsulated atoms arranged in a line could be set up with mutual spin orientations defining qbits to form the register of a quantum computer, the attraction being that such a system would be small and could be incorporated on a 'chip' rather than necessitating extensive atomic trapping and cooling methods.

What invites caution in attempting to extend Faraday screening to very small sizes is the 'skin effect' in electromagnetism, the scale size of which is frequency dependent. It is therefore pretty clear that the efficiency of screening must be frequency dependent and that, when the skin depth becomes comparable to atomic size, effects connected with the perfect conductor, such as Faraday screening, will no longer act in quite the same way as in classical electromagnetism.

This situation has been studied theoretically [34] and leads to the following picture. If an atom is trapped endohedrally inside a conducting sphere of nanoscale size, then this sphere will itself possess a Mie resonance [35] (in the case of a fullerene, this is simply the spectral feature called a 'giant resonance'). In other words, the charge distribution will set up an oscillation in response to an external electromagnetic field. In simple terms, the confining sphere acts like an antenna, and the encapsulated atom 'sits' within the field of this antenna.

At zero frequency, the charge distribution on the perfectly conducting sphere indeed adjusts itself so as to cancel the influence of external static fields within its volume. However, this does not remain as the frequency is raised and approaches the Mie resonance. To the contrary, when 
the frequency of the external radiation becomes Mie resonant, the metallic sphere turns into a miniature amplifier, and the atom placed inside is subjected to a strongly enhanced oscillating field. As the frequency of the incident radiation is raised still further and well above resonance, this enhancement fades away and, in fact, the screening effect disappears completely, giving place to full penetration of the volume inside the sphere. There is a simple function to describe this 'frequency dependent Faraday screening' which, again, is a typical nanoscale phenomenon and, not surprisingly, follows the shape of the Mie resonance of the sphere.

Thus, in applications involving screening on the nanoscale, it is important to take account of the frequency bath in which the system is placed.

An interesting consequence of this study relates to the observation of inner shell spectra of atoms and molecules. The reason for which the outer electronic shells of atoms do not prevent the excitation of inner shells by screening is basically the same: the X-rays exciting the system are of such high frequency that they penetrate the full atomic volume. However, it is only on the nanoscale that this extension of the Faraday screening principle acquires true significance. If, in place of a perfect conducting sphere, one has a fullerene molecule, in which the charge distribution is not evenly distributed over a spherical surface, then the effects described (both screening and anti-screening) will be less pronounced. However, they continue to dominate the response, as witnessed by the very existence of a giant resonance for such systems.

\section{Confinement and interacting resonances}

When atoms are confined in cavities of quantum size (which can happen on the nanoscale) then a variety of new effects can appear, depending on the symmetry of the confinement, its rigidity and the relative sizes of the atom and the confining cavity.

The simplest situation is rigid confinement at the centre of a spherical cavity. In this case, we expect to find a spectrum containing: (1) the resonances of the confined atom, (2) the resonances of the confining sphere and (3) the resonances of a spherical cavity of quantum size, or 'confinement resonances' [36]. Since the confined atom and host cavity form a single system, all these resonances interact and must obey the general rules of $\mathrm{K}$ matrix theory for any system of interacting particles [37] implying avoided crossings, symmetry reversals, width fluctuations, etc. as a result of the interactions.

However, many such effects will be hidden from view if the confinement is (1) off-centre and (2) not rigid, which is likely to occur if the force between the atom and the confining surface is attractive rather than repulsive and of the size of the confining cavity is significantly larger than the size of the confined atom. If the atom 'rattles' inside the confining sphere, then phase cancellation effects will tend to mask confinement resonances. In order to observe them clearly, it is therefore important to chose situations in which the confined atom is not free to move relative to the confining sphere, for example because their relative sizes are close to each other [38].

\section{Size and temperature}

Strictly speaking, a single temperature $T$ is defined for an ideal gas containing many particles, and this definition is the starting point in thermodynamics. It would, however, be extremely restrictive to stay only with this definition and with such ideal objects as the Black Body to make use of temperature, since there are so many other systems and situations for which a temperature is useful, even if the definition ceases to be quite so rigorous.

For example, we extend the temperature $T$ to an optically thin plasma (as in stellar atmospheres [39]) by introducing the Local Thermodynamic Equilibrium (LTE), in which case we allow the radiation emitted by the constituents to escape from the plasma without being reabsorbed. The radiation is, in this sense, outside the equilibrium processes of the plasma, although this does not prevent the radiation escaping from such a plasma conveying the most useful information about its condition. All we need to postulate is that the ions in this plasma possess a Maxwell-Boltzmann velocity distribution, from which we can infer an ion temperature $T_{i}$. Similarly, we can examine the Boltzmann distribution of electrons in the excited energy levels of the ions and the free electron distribution from the radiation continuum to define electron temperatures $T_{e}$, the only problem being that these various temperature do exist, but are not in equilibrium with each other. Multicomponent systems with different constituent temperatures are of course frequent in many branches of science. Molecular physicists are perfectly accustomed, for example, to analysing molecular spectra which define one temperature for electronic excitation, another for vibrational and yet another for rotational excitations.

Usually, such systems are of finite size, but large enough that one can gloss over the actual influence of size on the definitions of thermodynamic variables. However, Jellinek and Goldberg [40] have considered explicitly the relationship between statistical ensembles (paying special attention to the especially microcanonical ensemble) and have re-examined dynamics, the equipartition theorem, and the notion of a dynamical temperature placing the emphasis on finite size effects. They introduce a dynamical rather than a purely statistical approach, making no a priori assumptions about equiprobability and formulate as a postulate the dynamical equipartition ansatz, which allows them to define dynamical degrees of freedom (rather than taking ensemble averages) and then use these as their tools in the analysis.

The dynamical equipartition ansatz is a generalization of the usual 'equipartition theorem'. The difference between them is that this ansatz involves no a priori assumption of equal probability, whereas the more usual 'theorem' invoked in Statistical Mechanics does. The ansatz replaces the concept of equally probable kinematical degrees of freedom by the postulate that the time-averaged 
kinetic energy is equally distributed between dynamical degrees of freedom. The empirical basis for such a postulate is the intensive nature of thermodynamic temperature as a state variable. This approach prepares the way for axiomatic formulations of statistical mechanics and statistical analysis unencumbered by the assumption of equiprobability of the degrees of freedom. According to these authors, their novel formulation is 'ergodic' with regard to the actual dynamical behaviour of finite systems, irrespective of whether the latter are ordered or chaotic. In the special case where a system explores all the relevant dimensions of its configuration space with equal probability, the dynamical equipartition postulate becomes the traditional equipartition theorem and the dynamical degrees of freedom then coincide with the traditional kinematic degrees of freedom. Jellinek and Goldberg [40] have applied their approach and made use of dynamical degrees of freedom to analyse structural and phase-like transformations in atomic clusters.

There are even some implicit situations which carry the idea of using 'temperature' to intellectual extremes, well beyond the carefully argued situation of the previous example.

In statistical mechanics, from the ergodic principle, we assume that the average of a process parameter over time and its average over a statistical ensemble coincide. So, we can even imagine introducing a kind of electron 'temperature' for single atoms by turning a blind eye to the nature of the actual ensemble. Famously, Einstein [41] introduced a single two-level atom, placing it in equilibrium with a radiation field (Principle of Detailed Balance) to deduce its $A$ coefficient from the Black-Body radiation law. Today, we prefer to deduce $A$ from quantum field theory, but it was actually discovered by Einstein in this somewhat contorted way, with the further twist that the $A$ coefficient can only be defined for an isolated atom in the absence of external fields! Quite obviously, there exists no temperature for a single atom, and even less for an atom with only two levels, but this idealised argument of Einstein [41] proved of fundamental importance in Physics since it introduced for the first time the concept of spontaneous emission, which actually results from fluctuations of the vacuum in quantum field theory.

Clearly, a sensible equilibrium temperature must require many atoms before it can exist. But just how many are really needed to achieve a 'workable' temperature? Put in another way, if we create a chain of atoms, at what scale length will it begin to exhibit a proper thermodynamic temperature and genuine thermodynamic behaviour?

There have been a number of theoretical studies of this type of problem. For example, Ness et al. [42] use a Generalised Langevin Equation (GLE) scheme to study the thermal transport of low dimensional systems. In this GLE approach, the central classical region is connected to two realistic thermal baths kept at two different temperatures [43]. They model one-dimensional atomic chains connected to these three-dimensional baths. The thermal transport properties are studied as a function of the chain length and the temperature difference between the baths.
They obtain two different power laws are obtained for linear conductance versus chain length. For large temperatures and temperature differences, chains longer than 18 atoms present a diffusive transport regime with a temperature gradient across the system. For lower temperatures and temperature differences, they find a more ballistic-like regime. Their analysis suggests that the behaviour at higher temperature is mainly due to anharmonic effects within longer chains.

Again, with a transition occurring around 18 atoms in a chain, we are into the regime of nanophysics. It is perhaps also worth noting that, as one increases the size of a chain or cluster, we enter the range where a photon emitted from one part of the system can be re-absorbed in another, which is the first step towards thermal energy being transported from one part of a cluster to another. In other words, towards a system which would no longer be optically 'thin'.

Most physical systems in one way or another are anharmonic. For practical reasons, the vibrational density of states is usually modelled within the harmonic approximation, with some partial correction for anharmonicity. The reason is that the problem of an anharmonic densities of states has stubbornly resisted a general and exact solution, which would remain convenient and straightforward in its applications. However Jellinek and Aleinikava [44] have recently formulated such a solution within both classical and quantum mechanics. It is based on the actual dynamical behavior of systems as a function of energy, observed or monitored on a given time scale, which can be short or long. The resulting anharmonic densities of states are fully dynamically informed and, in general, time-dependent. As such, they lay the ground for the formulation of a new statistical mechanical framework that incorporating time, which is ergodic by construction, with respect to the actual dynamical behaviour of the system.

\section{Entropy and the direction of time}

Of course, in Thermodynamics, a genuine temperature does not come alone: when it emerges, entropy must also be present, as evidenced at the outset in the original formulation by Jellinek and Goldberg [40]. The latter property is extremely important, because the Entropy Principle is the only one in physics to determine the arrow of Time. This may seem a bit of an abstruse point, but could well prove a crucial issue relating to the discussion of the What is Life? [19] transition above, because the evolution of a living system can only occur in the direction of increasing time, so this direction must at least be definable for the system in question.

In this connection, systems which are highly symmetrical possess an intrinsic order which means that their entropy is essentially different from that of a less ordered system of similar size. We may therefore infer that the question of a What is Life? transition is not simply related to a size but must also be related to the degree of intrinsic symmetry. If it is too great, then it may prove 
difficult to establish a viable system, because, in this picture, excessive symmetry would itself act as a barrier to evolution.

\section{The Casimir effect on the nanoscale}

The Casimir effect [45] is the force between two uncharged conducting plates in close proximity to each other and (like the Einstein $A$ coefficient above) is a quantum field effect resulting from vacuum fluctuations. This force exists not only between parallel plates, but also between two conducting spheres [46] or a sphere and a plate.

To my knowledge, the Casimir force between metallic clusters, or between metallic clusters and a conducting surface has never been observed. The reason for including the effect in the present list is that the nanoscale range is the one in which conducting surfaces and planes first emerge from the atomic environment as the size of systems increases. Thus, a force between very closely spaced conductors is somehow a 'natural' situation to investigate on the nanoscale. Against this general idea, there are of course the usual difficulties in attempting such a measurement, an important one being that the conducting objects, whatever their shape, must be uncharged for the Casimir effect to appear.

Potentially, a scheme for detecting the Casimir force might involve studying 'nanobilliards' for mobile uncharged metallic clusters deposited on an insulating plane surface. This configuration clearly belongs to 'nanoscale dynamics'. The main problem in performing such an experiment would be that the Casimir force is extremely small compared to other forces in the system such as typical interactions between a cluster and a surface. The presence of a dielectric would also affect the outcome.

\section{Conclusion}

The present report describes no new results, nor is it intended as a comment on the validity of earlier results. Rather, it is hoped to summarise effects which are either (1) truly specific to the nanoscale or (2) should appear differently in the nanoscale range. In connection with DYSON, it is important to create such a list, in order to ensure that we are dealing with real science involving new phenomena and not merely with the repetition of well-known effects on a different scale. Naturally, the author makes no claim to have drawn up an exhaustive list. Rather, the intention is to spark off a process by which participants in this series of scientific meetings may establish a more complete and more fully researched series of open topics defining new directions in nanoscale dynamics.

The author thanks Professor Andrey Solov'yov for the opportunity to review the subject presented here at the DYSON meeting in Bad Ems and for numerous fruitful exchanges on all the subjects raised. Special thanks are due to Professor Julius Jellinek for illuminating discussions on size-dependent effects in Statistical Mechanics.

\section{Author contribution statement}

I am the sole author of this article and I take full responsibility for its contents.

Open Access This is an open access article distributed under the terms of the Creative Commons Attribution License (http://creativecommons.org/licenses/by/4.0), which permits unrestricted use, distribution, and reproduction in any medium, provided the original work is properly cited.

\section{References}

1. E. Merzbacher, Quantum Mechanics, 3rd edn. (John Wiley New Jersey, 1998), p. 672

2. R. Feynman, Lectures on Physics, Volume I to III (Addison Wesley Longman, 1970), p. 1552

3. J. Schwinger, Phys. Rev. 74, 92 (1948)

4. P. McEvoy, Niels Bohr: reflections on subject and object (Microanalytix, San Francisco, 2001), p. 523

5. B. Pascal, Les Pensées (Chez Lefèvre, Libraire, rue de l'Épernon, Paris, 1826)

6. A. Einstein, Relativity: The Special and General Theory, Authorised translation: R.W. Lawson (Methuen \& Co Ltd, London, 1916)

7. J.W. Gibbs, Elementary Principles of Statistical Mechanics (Dover Edition Paperback, 1960)

8. P.A.M. Dirac, The Principles of Quantum Mechanics, 4th edn. (Oxford at the Clarendon Press, 1962), p. 312

9. M.-N. Célérier, L. Nottale,, J. Phys. A: Math. Gen. 37, $931(2004)$

10. J.-Z. Zhang, Testing Spatial Noncommutativity via Rydberg Atoms, Phys. Rev. Lett. 93, 043002 (2004)

11. A. Einstein, The original contribution of Einstein was presented in 1917 at a meeting of the German Physical Society. A translation of the paper appears in The Collected Papers of Albert Einstein, A. Engel, Trans. (Princeton U. Press, Princeton, NJ, 1997), Vol. 6, p. 434

12. H. Poincaré, Acta Math. 38, 161 (1921)

13. W.R.S. Garton, F.S. Tomkins, Astrophys. J. 158, 839 (1969)

14. J.P. Connerade, G. Droungas, N.E. Karapanagioti, M.S. Zhang, J. Phys. B 30, 2047 (1997)

15. J.-P. Connerade, M.-S. Zhan, J. Rao, K.T. Taylor, J. Phys. B 32, 2351 (1999)

16. J.P. Connerade, K.T. Taylor, G. Droungas, N.E. Karapanagiati, M.S. Zhan, J. Rao, Atoms in crossed fields, 172nd WE Heraeus Seminar on Atoms and Molecules in Strong External Fields (Plenum Press, London and New York, 1998), pp. 121-134

17. A.M. Abdulla, S. Hogan, M.S. Zhan, J.-P. Connerade, J. Phys. B 37, L147 (2004)

18. G. Gamow, One Two Three ... Infinity: Facts and Speculations of Science (Viking, New York, 1961)

19. E. Schrödinger, What is Life? With Mind and Matter and Autobiographical Sketches, Foreword by Roger Penrose (Cambridge University Press, 1992)

20. H.J. Morowitz, American Sci. 99, 102 (2011)

21. L. Birgit, R.F. Kyle, C.W. Kelly, N.H. Hoi-Ying, B. Giovanni, C.T. Brian, A. Singh, H.W. Kenneth, C.E. Siegerist, S.G. Tringe, H.D. Kenneth, L.R. Comolli, J.F. Banfield, Nat. Commun. 6, 6372 (2015) 
22. J.-P. Connerade, A.V. Solov'yov, W. Greiner, Europhys. News 33, 200 (2002)

23. V.I. Arnold, Usp. Math. Nauk 18, 13 (1963)

24. V.I. Arnold, Russ. Math. Surv. 18, 9 (1963)

25. P. Debye, Ann. Phys. 39, 789 (1912)

26. C. Kittel, Introduction to solid state physics (John Wiley \& Sons, Inc., 1996)

27. A. Einstein, Ann. Phys. 22, 180 (1907)

28. M. Blackman, Proc. R. Soc. A 148, 390 (1935)

29. W.D. Knight, K. Clemenger, W.A. de Heer, W.A. Saunders, M.Y. Chou, M.L. Cohen, Phys. Rev. Lett. 52, $2141(1984)$

30. J.-P. Connerade, C. Bréchignac, J. Phys. B 27, 3759

31. S. Neukermans, E. Janssens, R.E. Silverans, P. Lievens, Magic numbers for shells of electrons and shells of atoms in binary clusters, Unpublished Report from Laboratorium voor Vaste-Stoffysica en Magnetisme, Katholieke Universiteit Leuven, Celestijnenlaan 200D, 3001 Leuven, Belgium (2006)

32. J. Jellinek, Transition from Atoms to Clusters to Condensed Matter, Advances in Chemical Physics, Proceedings of the 240 Conference Sciences Great Challenges, edited by A.R Dinner (John Wiley \& Sons, 2015), Vol. 157, p. 25
33. W. Harneit, Phys. Rev. A 65, 032322 (2002)

34. J.-P. Connerade, A.V. Solov'yov, J. Phys. B 38, 807 (2005)

35. G. Mie, Annalen der Phys., Vierte Folge, Band 25, 377 (1908)

36. J.-P. Connerade, V.K. Dolmatov, S.T. Manson, J. Phys. B 33, 2279 (2000)

37. J.-P. Connerade, in Highly Excited Atoms (Cambridge University Press, Cambridge, 2005), p. 501, Chap. 8

38. A.V. Solov'yov (Private Communication)

39. L. Aller, The Atmospheres of the Sun and Stars, 2nd edn. (Ronald Press Company, New York, 1963)

40. J. Jellinek, A. Goldberg, J. Chem. Phys. 113, 2570 (2000)

41. A. Einstein, Physikalische Zeitschrift, Band 18, 121 (1917)

42. H. Ness, L. Stella, C.D. Lorenz, L. Kantorovich, arXiv:1612.00990 (2016)

43. H. Ness, A. Genina, L. Stella C.D. Lorenz, L. Kantorovich, Phys. Rev. B 93, 174303 (2016)

44. J. Jellinek, D. Aleinikava, J. Chem. Phys. 144, 214103 (2016)

45. H.B.G. Casimir, Proc. Kon. Ned. Akad. Wetensch. B 51, 793 (1948)

46. L.P. Teo, arXiv:1503.07934v1 (2015) 